\title{
Are imported consumer products an important diffuse source of PFASs to the Norwegian environment?
}

\author{
Robin Vestergren ${ }^{a}{ }^{,}$, Dorte Herzke ${ }^{a}$, Thanh Wang ${ }^{b}$, Ian T. Cousins ${ }^{c}$ \\ ${ }^{a}$ Norwegian Institute for Air Research (NILU), 9296 Tromsø, Norway \\ b State Key Laboratory of Environmental Chemistry and Ecotoxicology, Research Center for Eco-Environmental Science, Chinese Academy of Sciences, \\ Beijing 100085, China \\ ${ }^{c}$ Department of Applied Environmental Science (ITM), Stockholm University, SE-106 91 Stockholm, Sweden
}

\section{A R T I C L E I N F O}

\section{Article history:}

Received 15 October 2014

Received in revised form

22 December 2014

Accepted 25 December 2014

Available online 30 January 2015

\section{Keywords:}

PFASs

PFOA

PFOS

FTOHs

Consumer products

Diffuse emissions

\begin{abstract}
A B S T R A C T
The aim of this study was to measure perfluoroalkyl substances in a selection of imported consumer products $(n=45)$ and estimate population normalized emission rates during the use phase. 6:2 and 8:2 fluorotelomer alcohol (FTOH) were found in the highest concentrations ranging from $<\mathrm{MDL}$ to 374 and $163 \mu \mathrm{g} \mathrm{m}^{-2}$ respectively. Concentrations of FTOHs were approximately 2-3 orders of magnitude higher than those of perfluoroalkyl carboxylic acids (PFCAs). Although perfluorooctane sulfonate (PFOS) was detected in one carpet sample at $1.7 \mu \mathrm{g} \mathrm{m}^{-2}$, the majority of samples complied with regulatory limits for PFOS in the EU. Population normalized emission rates of perfluorooctanoic acid, 6:2 FTOH and 8:2 FTOH from imported consumer products were estimated to be 6.6, 2130 and $197 \mu \mathrm{g} \mathrm{year}^{-1}$ capita $^{-1}$ respec- $^{-}$ tively for the "intermediate" emission scenario. The results from this study suggest that emissions from imported products would have a small impact on the environmental concentrations of perfluoroalkyl acids on a regional scale.
\end{abstract}

๑) 2015 Elsevier Ltd. All rights reserved.

\section{Introduction}

Per- and polyfluoroalkyl substances (PFASs) are a large group of synthetic chemicals which have been manufactured for their unique amphiphilic properties and chemical stability (Kissa, 2001). Over the past decade PFASs have received considerable attention as a new group of persistent environmental contaminants with global distribution (Lindstrom et al., 2011). Long-chain perfluoroalkyl carboxylic acids (PFCAs, $\mathrm{C}_{\mathrm{n}} \mathrm{F}_{2 \mathrm{n}+1} \mathrm{COOH}, \mathrm{n} \geq 7$ ) and perfluoroalkane sulfonic acids (PFSAs, $\mathrm{C}_{\mathrm{n}} \mathrm{F}_{2 \mathrm{n}+1} \mathrm{SO}_{3} \mathrm{H}, \mathrm{n} \geq 6$ ) are of special concern due to their persistence (Kissa, 2001; Natarajana et al., 2005), bioaccumulation potential (Conder et al., 2008) and toxicology (Lau et al., 2007). Concern regarding the hazard profile of PFCAs and PFSAs has led to a series of actions by regulators and the producing industry. In 2001, perfluorooctane sulfonate (PFOS) and related long-chain PFASs were phased out by the major global manufacturer in North America and Europe (3M Company, 2000; USEPA, 2000). Stewardship agreements have also been reached between the US Environmental Protection Agency, and leading global

\footnotetext{
* Corresponding author.

E-mail address: rv@nilu.no (R. Vestergren).
}

companies to reduce emissions and product content of perfluorooctanoic acid (PFOA) and related chemicals by $95 \%$ by 2010 and to work toward their elimination by 2015 (USEPA, 2006). PFOS has been included in the Stockholm Convention on Persistent Organic Pollutants as an Annex B substance, i.e., restricted in its use (UNEP, 2009) and more recently PFOA was identified as Substances of Very High Concern by the European Chemical Agency (ECHA, 2013).

The actions to reduce emissions of long-chain PFASs has lead to a substitution towards short-chain homologues which are considered to be less bioaccumulative (Conder et al., 2008) or PFASs with ether bonds inserted in the polyfluoroalkyl moiety which are perceived to be less persistent (Buck et al., 2011; Wang et al., 2013). However, a parallel trend is the continuous or increasing production of PFASs in other parts of the world than Europe and North America (Lindstrom et al., 2011; Lim et al., 2011; Xie et al., 2013). As Annex B of the Stockholm Convention has several exemptions for continuous use of PFOS, and other long-chain PFASs are still not recognized by international agreements (UNEP, 2009), these chemicals can still be used in similar applications as before the phase out actions took place. In China, the production of PFOS was estimated to increase from approximately 30 to 250 metric tons between 2001 and 2006 and has remained fairly constant over the 
period 2006-2011 (Xie et al., 2013). Production and use of PFCAs has also increased drastically in China, Russia and India over the past decade (Wang et al., 2014). The apparent success of phase-out actions in developed countries may, therefore, be described more correctly as a geographical shift in primary emission sources related to production and industrial use of PFASs (Wang et al., 2014). Yet, little is known about how diffuse sources of PFASs have been affected by the global trends in production and use.

The continuous production and use of PFOS and related chemicals indicates that import of consumer products may contribute to diffuse emissions of PFASs in countries where they are no longer produced. Release of unbound PFAS residuals from consumer products during the use phase has previously been identified as a source of PFCAs and PFSAs to the environment (Dinglasan-Panlilio and Mabury, 2006; Prevedouros et al., 2006; Paul et al., 2009) and a recent substance flow analysis performed in Switzerland reported that treated textiles and carpets (the majority from import) was a substantial source of PFOA in Switzerland (Buser and Morf, 2009). The textile industry in China has been identified as the primary industrial sector of fluorinated finishing agents with approximately 10000 metric tons of fluorinated finishing agents being used annually (Lim et al., 2011), but little is known about the abundance and composition of PFASs in Chinese finished products. Several monitoring studies have reported levels of residual PFASs in a variety of consumer products (Berger and Herzke, 2006; SFT, 2006; Norin and Schulze, 2007; Gewurtz et al., 2009; Herzke et al., 2012), but there are only a few studies specifically reporting the levels of PFAS residuals in consumer products of Chinese origin (Greenpeace, 2013; Brigden et al., 2013; Knepper et al., 2014).

The aim of this study was to explore the hypothesis that import of PFASs in consumer products from China appreciably adds to the environmental emissions in non-producing countries using Norway as a case study. China is the main origin of textiles purchased in Norway, contributing $40 \%$ of the overall textile import into Norway in 2007 (SSB, 2008/48). Furthermore, China is one of the countries with on-going production of long-chain PFASs (Wang et al., 2014). Thus, textiles, carpets, clothing, and food contact materials imported from China to Norway were specifically investigated to (i) measure extractable PFAS residuals in imported consumer products of Chinese origin, (ii) evaluate compliance with regulatory guidelines for PFAS content in consumer products and (iii) estimate the resulting emissions to air and water from the investigated product categories.

\section{Methods}

\subsection{Sample collection}

A total of 45 furniture textile, carpet, clothing and food contact material samples were purchased from three major retail stores in Tromsø and Trondheim during the period November 2012-February 2013 (Table S1). The sampling campaign was designed to screen for PFASs in a wide range of consumer products belonging to product categories which have previously been found to contain PFAS residuals (Berger and Herzke, 2006; SFT, 2006; Norin and Schulze, 2007; Gewurtz et al., 2009; Washburn et al., 2005; Begley et al., 2005; Herzke et al., 2012). A second selection criteria for the purchased product categories was that they were representative of products imported from China to Norway in large quantities (SSB, 2013). The individual products were randomly selected from a broad range of products without any prior knowledge of surface treatment with PFASs in order to create a representative set of samples corresponding to the volumes of goods being imported to Norway from China. Outdoor clothing were excluded from the selection due to recent studies on these types of consumer products (Greenpeace, 2013; Brigden et al., 2013; Knepper et al., 2014). For this product category, concentration data from previous reports were used to calculate population normalized emission rates (see Section 2.3).

\subsection{Chemical analysis}

\subsubsection{Chemicals}

The 22 target analytes in this study were $C_{4}-C_{14}$ PFCAs, $C_{4}, C_{6}$, $C_{8}, C_{10}$ PFSAs, 4:2, 6:2, 8:2 and 10:2 FTOHs, N-ethyl perfluorooctane sulfonamidoethanol (EtFOSE), N-Methyl perfluorooctane sulfonamidoethanol (MeFOSE), N-Ethyl perfluorooctane sulfonamide (EtFOSA) and N-Methyl perfluorooctane sulfonamide (MeFOSA). Mass-labeled internal standards were ${ }^{13} \mathrm{C}_{4}-\mathrm{PFCAs},{ }^{18} \mathrm{O}_{2}$-PFSAs ${ }^{13} \mathrm{C}_{4}$-FTOHs, D9-EtFOSE, $\mathrm{D}_{7}$-MeFOSE, $\mathrm{D}_{5}$-EtFOSA and $\mathrm{D}_{3}-\mathrm{MeFOSA}$ (see also the supplementary material). 3,7-branched perfluorodecanoate (3,7 DMPFDOA) and 7:1 FTOH were used as volumetric standards to calculate the recovery of anionic and neutral PFASs respectively. All native and isotope labeled PFCA and PFSA standard compounds were purchased from Wellington Laboratories (Guelph, ON, Canada) in $2 \mu \mathrm{g} \mathrm{mL}^{-1}$ solution mixtures. All solvents and reagents used in this work were of LiChrosolv grade, and were purchased from Merck-Schuchardt (Hohenbrunn, Germany).

\subsubsection{Extraction and analysis}

Two previously reported methods were used to extract and analyze ionic PFASs (PFCAs and PFSAs) and neutral PFASs (FOSEs, FOSAs and FTOHs) separately (Herzke et al., 2012). Briefly, two squares $(10 \times 10 \mathrm{~cm})$ of textile, carpet or food contact material were cut with a pair of scissors which had been rinsed in methanol. The sample was weighed, cut into small pieces and spiked with masslabeled internal standards. Samples were extracted with methanol for ionic compounds and ethyl acetate for neutral PFASs two times for $15 \mathrm{~min}$ in an ultrasonic bath with vortex treatment in between. After centrifugation and solvent evaporation, an aliquot of $1 \mathrm{~mL}$ extract was transferred for dispersive clean up with ENVI-Carb (50 mg, $1 \mathrm{~mL}, 100-400$ mesh, Supelco, USA) according to the method of Powley et al. (2005). Addition of glacial acetic acid ( $50 \mu \mathrm{l}$ ) to the ENVI-carb was used only for the ionic PFASs. Finally, a volume of $500 \mu \mathrm{L}$ of the supernatant was transferred to a new glass vial and $2 \mathrm{ng}$ of the volumetric standard was added and mixed thoroughly. For ionic PFASs, aliquots of $100 \mu \mathrm{L}$ were diluted with $2 \mathrm{mM}$ aqueous ammonium acetate solution $\left(\mathrm{NH}_{4} \mathrm{OAc}, 50 / 50, \mathrm{v} / \mathrm{v}\right)$ prior to analysis whereas neutral PFASs were analyzed in ethyl acetate. PFCAs and PFSAs were analyzed by ultra-high performance liquid chromatography triple-quadrupole mass-spectrometry (UPLC-MS/MS) whereas FOSEs, FOSAs and FTOHs were analyzed by gas chromatography-mass-spectrometry (GC-MS) in selected ion monitoring (SIM)-mode. Further details on the instrumental analysis are given in the supplementary material.

\subsubsection{Quality assurance}

The analytical method has previously been used for determination of extractable residuals in textiles (Berger and Herzke, 2006; Herzke et al., 2012). The NILU Tromsø laboratory has contributed in a number of inter-laboratory comparison studies for PFAS analysis with satisfactory results (Weiss et al., 2013). Recoveries of internal standards, procedural and instrumental blanks and method detection limits are regularly monitored as quality criteria for the analysis. Average recoveries for the internal standards ranged from 46 to $108 \%$ and 62 to $143 \%$ for ionic and neutral PFASs respectively (see also Table S2-S3). The recoveries indicated satisfactory extraction efficiency and only limited losses during sample preparation for the majority of analytes, whereas recoveries $>100 \%$ for 
some analytes also indicated a slight matrix enhancement of the ionization efficiency of the volumetric standards compared to the internal standards. A commonly observed problem in trace analysis of PFASs is contamination from the extraction and clean-up procedures. To circumvent this problem solvents and disposable laboratory equipment were routinely checked for PFSA contamination prior to use. Three procedural blanks were also included for every batch of 20 samples and 1 solvent injection blank was analyzed for every 10 samples injected. No blank contamination was detected, however, for any of the analytes. Within sample replicates were performed for 6 samples with low $(<50 \%)$ recovery for one or several of the analytes which were extracted and analyzed in triplicate (Table S4-S5). Method detection limits (MDLs) were calculated on the basis of the lowest calibration standard giving a signal-to-noise ratio of 3 and the concentration factor of the extraction and clean up procedure. No significant differences in background noise were observed between the different samples; thus general MDLs were derived for all consumer product samples based on worst case calculations assuming 60\% total average recovery. The MDLs for individual substances ranged from 0.005 to $0.010 \mu \mathrm{g} \mathrm{m}^{-2}$ and 0.5 to $2 \mu \mathrm{g} \mathrm{m}^{-2}$ for ionic and neutral PFASs respectively.

\subsection{Calculation of population normalized emission rates from imported consumer products}

The methodology to calculate population normalized emission rates $\left(E R_{a, w}, \mu \mathrm{g} \mathrm{year}^{-1}\right.$ capita $\left.^{-1}\right)$ of PFASs to air and water respectively from imported consumer products was based on the following equation:

$E R_{a, w}=\sum_{i=1}^{n} \frac{C_{i}^{*} Q_{i}^{*} E F_{a, w}}{P}$

where $C_{i}$ is the concentration of extractable PFASs in a specific consumer product category $\left(\mu \mathrm{g} \mathrm{kg}^{-1}\right), Q_{i}$ is the quantity of that consumer product category being imported from China to Norway from China $\left(\mathrm{kg} \mathrm{year}^{-1}\right), E F_{a, w}$ is the emission factor (unitless) describing the transfer efficiency of different PFASs to air and water respectively and $P$ is the population of Norway (capita). Extractable concentrations in the consumer product categories were derived from the chemical analysis (described above) with the exception of outdoor clothing. For these products, a data set of 17 measurements were derived from recently analyzed products (2012-2013) manufactured in China (Greenpeace, 2013; Brigden et al., 2013; Knepper et al., 2014). Although the concentrations of PFASs in this paper are reported in $\mu \mathrm{g} \mathrm{m}^{-2}$, transformation to $\mu \mathrm{g} \mathrm{kg}^{-1}$ was performed by using the weight per surface area of consumer products (Table S1) to enable calculation of emission rates from import quantities. The quantities of different consumer product categories imported from China and population of Norway were derived from publicly available national statistics in Norway (SSB, 2013). The summed annual import of furniture textiles, carpets/mats, cotton and leather clothing and food packaging material and outdoor clothing was estimated to be 3920, 367, $10700,628,11600$ metric tons respectively. For a more comprehensive summary of the import statistics see Table S6-S10 of the Supplementary Material. Emission factors of different PFASs were derived from a recent study conducting washing and off-gassing experiments with outdoor jackets treated with PFASs (Knepper et al., 2014). The study of Knepper et al. demonstrated that extractable residuals of PFCAs and PFSAs were completely removed in the first wash of the textile under typical machine wash conditions (Knepper et al., 2014). In our calculations we assumed that $100 \%$ of the extractable PFCA and
PFSA residuals in furniture textiles, food packaging, carpets and clothing is emitted during washing to municipal wastewater within a year of usage. In contrast to PFCAs and PFSAs, neutral PFASs (including FTOHs) typically have a low water solubility and a high vapor pressure (Kissa, 2001). A flow-through study showed that a significant part of the extractable FTOHs present in jackets was released to air during 5-days of forced ventilation (Knepper et al., 2014). The fraction of extractable chemical that was released to air was $53.7 \%$ and $12.4 \%$ for $6: 2$ FTOH and $8: 2$ FTOH respectively. During a period of one year of repeated use it may, however, be expected that a larger fraction of the FTOHs are released compared to five days of forced ventilation. For calculation of emissions of FTOHs from consumer products to air, we therefore used 95th percentile values (100\% and $17.6 \%$ respectively) from the flowthrough study (Knepper et al., 2014). The variability in concentrations within the consumer product categories were used to estimate the uncertainty range in population normalized emission rates. A low, intermediate and high scenario corresponding to the 5 th, $50^{\text {th }}$ and 95 th percentile concentrations present in the products was generated to reflect the uncertainty range in emissions (see Tables S11-S12).

\section{Results and discussion}

\subsection{Concentrations of extractable PFASs in consumer products}

Overall, PFASs were detected in $69 \%$ of the samples with $6: 2$ FTOH, 8:2 FTOH and PFOA being the most frequently detected analytes. The extractable concentrations of PFCAs and FTOHs are shown in Tables 1 and 2. PFSAs were only detected in two samples (Table S13) whereas FOSEs and FOSAs were not detected in any of the products. The concentrations of individual target analytes displayed a large variation within and between the different consumer product groups.

The highest concentrations among the furniture textile samples were observed in the down quilt (T33), sofa pillow fabric (T23) and two curtain samples (T2 and J3) which contained individual PFCAs in the range $0.14-1.022 \mu \mathrm{g} \mathrm{m}^{-2}$. Although PFOA was the most frequently detected homologue, PFHpA was detected in the highest concentration. For all other furniture textiles, PFCAs and PFSAs were present at trace concentrations between MDL and $0.043 \mu \mathrm{g} \mathrm{m}^{-2}$. The curtain sample (T2) also had relatively high concentrations of $8: 2 \mathrm{FTOH}\left(164 \mu \mathrm{g} \mathrm{m}^{-2}\right)$ and 10:2 FTOH $\left(60 \mu \mathrm{g} \mathrm{m}^{-2}\right)$ while $6: 2$ FOTH was detected at relatively high concentrations in one pillow $\left(\mathrm{J} 1 ; 374 \mu \mathrm{g} \mathrm{m}^{-2}\right)$, one pillow case (T4; $242 \mu \mathrm{g} \mathrm{m}^{-2}$ ), one towel (T5; $70 \mu \mathrm{g} \mathrm{m}^{-2}$ ), one chair cover (T11; $26 \mu \mathrm{g} \mathrm{m}^{-2}$ ), two fleece blankets (T12 and J6; 18 and $30 \mu \mathrm{g} \mathrm{m}^{-2}$ ), one seat pillow $\left(\mathrm{J} 4 ; 43 \mu \mathrm{g} \mathrm{m}^{-2}\right.$ ) and one curtain sample (J3; $54 \mu \mathrm{g} \mathrm{m}^{-2}$ ). In carpet and mat samples, 6:2 FTOH was the most frequently detected analyte ranging between MDL and $74 \mu \mathrm{g} \mathrm{m}^{-2}$ (median of $25 \mu \mathrm{g} \mathrm{m}^{-2}$ ). Concentrations of 4:2 FTOH and 8:2 FTOH close to the MDL were typically found in the same samples which contained 6:2 FTOH. PFOS was also detected in one carpet sample at $1.7 \mu \mathrm{g} \mathrm{m}^{-2}$. PFASs were detected in all cotton and leather clothing (including one imitation leather jacket). However, the concentrations of extractable PFASs were generally close to the MDL, except for one cotton bath robe sample (J2) which contained $370 \mu \mathrm{g} \mathrm{m}^{-2}$ of $6: 2 \mathrm{FTOH}$. All food contact material samples contained detectable concentrations of PFCAs; one paper plate (T26) and one paper cup (T29) sample contained elevated concentrations of PFOA $\left(0.5 \mu \mathrm{g} \mathrm{m}^{-2}\right) .8: 2 \mathrm{FTOH}$ was also detected in three of the food contact material samples, ranging up to $12.3 \mu \mathrm{g} \mathrm{m}^{-2}$.

The primary use of PFASs in furniture textiles, carpets, mats and clothing is to impart water and stain repellency. Although the active coating used for surface treatment are typically polymeric 
Table 1

Extractable concentrations of PFCAs $\left(\mu \mathrm{g} \mathrm{m}^{-2}\right.$ ) in consumer products imported from China.

\begin{tabular}{|c|c|c|c|c|c|c|c|c|c|c|c|c|c|}
\hline \multirow[t]{2}{*}{ Sample nr. } & \multirow[t]{2}{*}{ Sample category } & \multirow[t]{2}{*}{ Sample type } & \multicolumn{11}{|c|}{ Concentration $\left(\mu \mathrm{g} \mathrm{m}^{-2}\right)$} \\
\hline & & & PFBA & PFPA & PFHxA & PFHpA & PFOA & PFNA & PFDA & PFUnDA & PFDoDA & PFTrDA & PFTeDA \\
\hline $\mathrm{T} 1$ & Furniture textiles & Table cloth & $<0.01$ & $<0.005$ & $<0.005$ & $<0.005$ & $<0.005$ & $<0.005$ & $<0.005$ & $<0.005$ & $<0.005$ & $<0.005$ & $<0.005$ \\
\hline $\mathrm{T} 2$ & Furniture textiles & Curtain & $<0.01$ & 0.246 & 0.812 & 1.022 & 0.914 & 0.097 & 0.425 & 0.051 & 0.247 & $<0.005$ & $<0.005$ \\
\hline T3 & Furniture textiles & Pillow case & $<0.01$ & $<0.005$ & $<0.005$ & $<0.005$ & $<0.005$ & $<0.005$ & $<0.005$ & $<0.005$ & $<0.005$ & $<0.005$ & $<0.005$ \\
\hline $\mathrm{T} 4$ & Furniture textiles & Pillow case & $<0.01$ & $<0.005$ & 0.010 & $<0.005$ & 0.007 & $<0.005$ & $<0.005$ & $<0.005$ & $<0.005$ & $<0.005$ & $<0.005$ \\
\hline T5 & Furniture textiles & Towel & $<0.01$ & $<0.005$ & $<0.005$ & $<0.005$ & 0.014 & $<0.005$ & $<0.005$ & $<0.005$ & $<0.005$ & $<0.005$ & $<0.005$ \\
\hline T6 & Furniture textiles & Bed sheet & $<0.01$ & $<0.005$ & 0.006 & $<0.005$ & 0.009 & $<0.005$ & $<0.005$ & $<0.005$ & $<0.005$ & $<0.005$ & $<0.005$ \\
\hline $\mathrm{T} 7$ & Furniture textiles & Baby blanket & $<0.01$ & $<0.005$ & $<0.005$ & $<0.005$ & $<0.005$ & $<0.005$ & $<0.005$ & $<0.005$ & $<0.005$ & $<0.005$ & $<0.005$ \\
\hline T8 & Furniture textiles & Chair cover & $<0.01$ & $<0.005$ & $<0.005$ & $<0.005$ & 0.023 & $<0.005$ & $<0.005$ & $<0.005$ & $<0.005$ & $<0.005$ & $<0.005$ \\
\hline T9 & Furniture textiles & Kitchen towel & $<0.01$ & $<0.005$ & $<0.005$ & $<0.005$ & $<0.005$ & $<0.005$ & $<0.005$ & $<0.005$ & $<0.005$ & $<0.005$ & $<0.005$ \\
\hline $\mathrm{T} 10$ & Furniture textiles & Baby blanket & $<0.01$ & $<0.005$ & 0.006 & $<0.005$ & $<0.005$ & $<0.005$ & $<0.005$ & $<0.005$ & $<0.005$ & $<0.005$ & $<0.005$ \\
\hline T11 & Furniture textiles & Chair cover & $<0.01$ & $<0.005$ & $<0.005$ & $<0.005$ & $<0.005$ & $<0.005$ & $<0.005$ & $<0.005$ & $<0.005$ & $<0.005$ & $<0.005$ \\
\hline T12 & Furniture textiles & Fleece blanket & $<0.01$ & $<0.005$ & 0.015 & $<0.005$ & 0.010 & $<0.005$ & $<0.005$ & $<0.005$ & $<0.005$ & $<0.005$ & $<0.005$ \\
\hline T22 & Furniture textiles & Sofa cover & $<0.01$ & $<0.005$ & $<0.005$ & $<0.005$ & $<0.005$ & $<0.005$ & $<0.005$ & $<0.005$ & $<0.005$ & $<0.005$ & $<0.005$ \\
\hline $\mathrm{T} 23$ & Furniture textiles & Sofa pillow fabric & $<0.01$ & $<0.005$ & 0.066 & 0.089 & 0.140 & 0.048 & 0.024 & $<0.005$ & 0.022 & $<0.005$ & $<0.005$ \\
\hline $\mathrm{T} 24$ & Furniture textiles & Roll packed quilts & $<0.01$ & $<0.005$ & 0.011 & $<0.005$ & $<0.005$ & $<0.005$ & $<0.005$ & $<0.005$ & $<0.005$ & $<0.005$ & $<0.005$ \\
\hline Т30 & Furniture textiles & Baby bed cover & $<0.01$ & $<0.005$ & $<0.005$ & $<0.005$ & 0.016 & $<0.005$ & 0.010 & $<0.005$ & 0.005 & $<0.005$ & $<0.005$ \\
\hline T31 & Furniture textiles & Baby mattress & $<0.01$ & $<0.005$ & $<0.005$ & $<0.005$ & 0.011 & $<0.005$ & $<0.005$ & $<0.005$ & $<0.005$ & $<0.005$ & $<0.005$ \\
\hline T32 & Furniture textiles & Down quilt & $<0.01$ & $<0.005$ & $<0.005$ & $<0.005$ & $<0.005$ & $<0.005$ & $<0.005$ & $<0.005$ & $<0.005$ & $<0.005$ & $<0.005$ \\
\hline Т33 & Furniture textiles & Down quilt & $<0.01$ & $<0.005$ & 0.023 & 0.027 & 0.060 & 0.030 & 0.055 & 0.024 & 0.063 & 0.008 & $<0.005$ \\
\hline J1 & Furniture textiles & Pillow & $<0.01$ & $<0.005$ & $<0.005$ & $<0.005$ & $<0.005$ & $<0.005$ & $<0.005$ & $<0.005$ & $<0.005$ & $<0.005$ & $<0.005$ \\
\hline $\mathrm{J} 3$ & Furniture textiles & Curtain & $<0.01$ & $<0.005$ & 0.010 & $<0.005$ & 0.501 & $<0.005$ & $<0.005$ & $<0.005$ & $<0.005$ & $<0.005$ & $<0.005$ \\
\hline $\mathrm{J} 4$ & Furniture textiles & Seat pillow & $<0.01$ & $<0.005$ & $<0.005$ & 0.006 & 0.011 & 0.014 & $<0.005$ & $<0.005$ & $<0.005$ & $<0.005$ & $<0.005$ \\
\hline J6 & Furniture textiles & Fleece blanket & $<0.01$ & $<0.005$ & $<0.005$ & $<0.005$ & $<0.005$ & $<0.005$ & $<0.005$ & $<0.005$ & $<0.005$ & $<0.005$ & $<0.005$ \\
\hline J7 & Furniture textiles & Bed linen & $<0.01$ & $<0.005$ & 0.043 & 0.010 & 0.034 & $<0.005$ & 0.016 & $<0.005$ & $<0.005$ & $<0.005$ & $<0.005$ \\
\hline $\mathrm{J} 8$ & Furniture textiles & Towel & $<0.01$ & $<0.005$ & $<0.005$ & $<0.005$ & $<0.005$ & $<0.005$ & $<0.005$ & $<0.005$ & $<0.005$ & $<0.005$ & $<0.005$ \\
\hline $\mathrm{J} 10$ & Furniture textiles & Quilt & $<0.01$ & $<0.005$ & $<0.005$ & $<0.005$ & $<0.005$ & $<0.005$ & $<0.005$ & $<0.005$ & $<0.005$ & $<0.005$ & $<0.005$ \\
\hline $\mathrm{J} 12$ & Furniture textiles & Quilt & $<0.01$ & $<0.005$ & $<0.005$ & $<0.005$ & $<0.005$ & $<0.005$ & $<0.005$ & $<0.005$ & $<0.005$ & $<0.005$ & $<0.005$ \\
\hline T13 & Carpets/mats & Sheep skin & $<0.01$ & $<0.005$ & 0.014 & $<0.005$ & 0.014 & $<0.005$ & $<0.005$ & $<0.005$ & $<0.005$ & $<0.005$ & $<0.005$ \\
\hline T17 & Carpets/mats & Carpet & $<0.01$ & $<0.005$ & 0.039 & 0.007 & 0.029 & 0.006 & 0.014 & $<0.005$ & $<0.005$ & $<0.005$ & $<0.005$ \\
\hline T18 & Carpets/mats & Carpet & $<0.01$ & $<0.005$ & $<0.005$ & $<0.005$ & 0.005 & $<0.005$ & $<0.005$ & $<0.005$ & $<0.005$ & $<0.005$ & $<0.005$ \\
\hline T19 & Carpets/mats & Carpet & $<0.01$ & $<0.005$ & $<0.005$ & $<0.005$ & $<0.005$ & $<0.005$ & $<0.005$ & $<0.005$ & $<0.005$ & $<0.005$ & $<0.005$ \\
\hline T20 & Carpets/mats & Sheep skin & $<0.01$ & $<0.005$ & 0.153 & 0.052 & 0.350 & 0.077 & 0.076 & $<0.005$ & $<0.005$ & $<0.005$ & $<0.005$ \\
\hline $\mathrm{T} 21$ & Carpets/mats & Rug & $<0.01$ & $<0.005$ & 0.040 & $<0.005$ & 0.059 & 0.029 & 0.022 & $<0.005$ & $<0.005$ & $<0.005$ & $<0.005$ \\
\hline J5 & Carpets/mats & Lamb skin & $<0.01$ & $<0.005$ & 0.052 & $<0.005$ & $<0.005$ & $<0.005$ & $<0.005$ & $<0.005$ & $<0.005$ & $<0.005$ & $<0.005$ \\
\hline J9 & Carpets/mats & Bath mat & $<0.01$ & $<0.005$ & $<0.005$ & $<0.005$ & $<0.005$ & $<0.005$ & $<0.005$ & $<0.005$ & $<0.005$ & $<0.005$ & $<0.005$ \\
\hline $\mathrm{J} 11$ & Carpets/mats & Bath mat & $<0.01$ & $<0.005$ & $<0.005$ & $<0.005$ & 0.083 & $<0.005$ & $<0.005$ & $<0.005$ & $<0.005$ & $<0.005$ & $<0.005$ \\
\hline T14 & Cotton/leather clothes & Jacket & $<0.01$ & $<0.005$ & $<0.005$ & 0.008 & 0.087 & 0.006 & $<0.005$ & $<0.005$ & $<0.005$ & $<0.005$ & $<0.005$ \\
\hline T15 & Cotton/leather clothes & Jacket & $<0.01$ & $<0.005$ & $<0.005$ & $<0.005$ & 0.019 & 0.008 & $<0.005$ & $<0.005$ & $<0.005$ & $<0.005$ & $<0.005$ \\
\hline T16 & Cotton/leather clothes & Jacket & $<0.01$ & $<0.005$ & $<0.005$ & $<0.005$ & 0.018 & $<0.005$ & $<0.005$ & $<0.005$ & $<0.005$ & $<0.005$ & $<0.005$ \\
\hline $\mathrm{J} 2$ & Cotton/leather clothes & Bath robe & $<0.01$ & $<0.005$ & $<0.005$ & $<0.005$ & $<0.005$ & $<0.005$ & $<0.005$ & $<0.005$ & $<0.005$ & $<0.005$ & $<0.005$ \\
\hline $\mathrm{T} 25$ & Food contact material & Baking mold & $<0.01$ & $<0.005$ & $<0.005$ & $<0.005$ & $<0.005$ & $<0.005$ & $<0.005$ & $<0.005$ & $<0.005$ & $<0.005$ & $<0.005$ \\
\hline $\mathrm{T} 26$ & Food contact material & Paper plates & $<0.01$ & $<0.005$ & 0.010 & 0.022 & 0.496 & 0.022 & 0.016 & $<0.005$ & $<0.005$ & $<0.005$ & $<0.005$ \\
\hline $\mathrm{T} 27$ & Food contact material & Baking cover & $<0.01$ & $<0.005$ & 0.024 & $<0.005$ & 0.026 & $<0.005$ & $<0.005$ & $<0.005$ & $<0.005$ & $<0.005$ & $<0.005$ \\
\hline T28 & Food contact material & Paper plates & $<0.01$ & $<0.005$ & $<0.005$ & $<0.005$ & 0.071 & $<0.005$ & $<0.005$ & $<0.005$ & $<0.005$ & $<0.005$ & $<0.005$ \\
\hline T29 & Food contact material & Paper cup & $<0.01$ & $<0.005$ & $<0.005$ & 0.023 & 0.051 & 0.030 & 0.043 & 0.038 & 0.040 & 0.034 & $<0.005$ \\
\hline
\end{tabular}

Concentrations above MDL are presented in bold.

materials (Buck et al., 2011), low molecular weight PFASs, including FTOHs and PFCAs, may be present as unreacted starting materials or impurities from the production process (Dinglasan-Panlilio and Mabury, 2006; Washburn et al., 2005; Mawn et al., 2005; Larsen et al., 2006; Russell et al., 2008; Russell et al., 2010). The variable concentrations of PFCAs and PFSAs and FTOHs most likely reflect the degree of treatment with side-chain fluorinated polymers to provide surface protection (Dinglasan-Panlilio and Mabury, 2006; Buck et al., 2011). The low trace-level concentrations of PFCAs in the majority of samples may, however, also be due to a low level addition of PFCAs to products to act as leveling agents in dyes and detergents (Kissa, 2001). It is also possible that products that have not been intentionally treated with PFASs become contaminated throughout the retail chain as it has been shown that furniture and outdoor textile shops have highly elevated concentrations of FTOHs compared to other indoor environments (Schlummer et al., 2013). The presence of low molecular weight PFASs in food contact materials has previously been attributed to residuals present in polyfluoroalkyl phosphoric acid esters (PAPs) which are used for the treatment of paper and board (Begley et al., 2005; Trier et al., 2011).
3.2. Comparison of PFAS concentrations with previous studies and regulatory guidelines

The present study demonstrates that PFASs can be detected in a wide range of imported consumer products using sensitive analytical techniques. However, among the 45 products analyzed, the majority of samples contained low molecular weight, nonpolymeric PFASs at trace levels. The presence of PFASs was not due to intentional treatment of textiles with these substances, but rather due to residual impurities of low molecular weight PFASs in polymeric surface treatments that are based on PFAS chemistries (Paul et al., 2009; Wang et al., 2013). One out of 45 samples contained concentrations of PFOS $\left(1.7 \mu \mathrm{g} \mathrm{m}^{-2}\right)$ above the EU and Norwegian limit of $1 \mu \mathrm{g} \mathrm{m}^{-2}$ (ECHA 2013). To put these measurements into perspective a comparison with previous studies on PFASs residuals in various consumer products is presented in Table 3.

The literature on PFASs in consumer products consists of industry studies conducted on fluorochemical raw materials or treated products with a known composition (Fraunhofer, 2004; 
Table 2

Extractable concentrations of neutral PFASs $\left(\mu \mathrm{g} \mathrm{m}^{-2}\right)$ in consumer products imported from China.

\begin{tabular}{|c|c|c|c|c|c|c|}
\hline \multirow{3}{*}{$\begin{array}{l}\text { Sample } \\
\text { nr. }\end{array}$} & \multirow{3}{*}{ Sample category } & \multirow[t]{3}{*}{ Sample type } & \multicolumn{4}{|c|}{ Concentration $\left(\mu \mathrm{g} \mathrm{m}^{-2}\right)$} \\
\hline & & & $4: 2$ & $6: 2$ & $8: 2$ & $10: 2$ \\
\hline & & & FTOH & FTOH & FTOH & FTOH \\
\hline $\mathrm{T} 1$ & Furniture textiles & Table cloth & $<0.5$ & 2.23 & 0.9 & $<0.5$ \\
\hline $\mathrm{T} 2$ & Furniture textiles & Curtain & $<0.5$ & 8.49 & 163 & 60.17 \\
\hline T3 & Furniture textiles & Pillow case & $<0.5$ & $<0.5$ & 1.0 & $<0.5$ \\
\hline $\mathrm{T} 4$ & Furniture textiles & Pillow case & $<0.5$ & 241.1 & 1.4 & $<0.5$ \\
\hline T5 & Furniture textiles & Towel & $<0.5$ & 69.99 & 1.3 & 0.87 \\
\hline T6 & Furniture textiles & Bed sheet & $<0.5$ & $<0.5$ & 0.7 & $<0.5$ \\
\hline $\mathrm{T} 7$ & Furniture textiles & baby blanket & $<0.5$ & $<0.5$ & $<0.5$ & $<0.5$ \\
\hline T8 & Furniture textiles & chair cover & $<0.5$ & 0.55 & $<0.5$ & $<0.5$ \\
\hline T9 & Furniture textiles & Kitchen towel & $<0.5$ & $<0.5$ & $<0.5$ & $<0.5$ \\
\hline T10 & Furniture textiles & Baby blanket & $<0.5$ & $<0.5$ & $<0.5$ & $<0.5$ \\
\hline T11 & Furniture textiles & chair cover & $<0.5$ & 26.2 & 1.0 & 0.55 \\
\hline T12 & Furniture textiles & fleece blanket & $<0.5$ & 18.2 & 2.4 & 0.98 \\
\hline $\mathrm{T} 22$ & Furniture textiles & Sofa cover & $<0.5$ & $<0.5$ & $<0.5$ & $<0.5$ \\
\hline $\mathrm{T} 23$ & Furniture textiles & Sofa pillow fabric & $<0.5$ & $<0.5$ & $<0.5$ & $<0.5$ \\
\hline $\mathrm{T} 24$ & Furniture textiles & Roll packed quilts & $<0.5$ & $<0.5$ & $<0.5$ & $<0.5$ \\
\hline T30 & Furniture textiles & Baby bed cover & $<0.5$ & $<0.5$ & $<0.5$ & $<0.5$ \\
\hline T31 & Furniture textiles & Baby mattress & $<0.5$ & $<0.5$ & $<0.5$ & $<0.5$ \\
\hline T32 & Furniture textiles & Down quilt & $<0.5$ & $<0.5$ & $<0.5$ & $<0.5$ \\
\hline T33 & Furniture textiles & Down quilt & $<0.5$ & $<0.5$ & $<0.5$ & $<0.5$ \\
\hline J1 & Furniture textiles & Pillow & $<0.5$ & 373.7 & $<0.5$ & $<0.5$ \\
\hline $\mathrm{J} 3$ & Furniture textiles & Curtain & $<0.5$ & 54.0 & $<0.5$ & $<0.5$ \\
\hline $\mathrm{J} 4$ & Furniture textiles & Seat pillow & $<0.5$ & 43.4 & $<0.5$ & $<0.5$ \\
\hline J6 & Furniture textiles & Fleece blanket & $<0.5$ & 29.6 & $<0.5$ & $<0.5$ \\
\hline $\mathrm{J} 7$ & Furniture textiles & Bed linen & $<0.5$ & $<0.5$ & $<0.5$ & $<0.5$ \\
\hline J8 & Furniture textiles & Towel & $<0.5$ & 36.1 & $<0.5$ & $<0.5$ \\
\hline $\mathrm{J} 10$ & Furniture textiles & Quilt & $<0.5$ & 16.8 & $<0.5$ & $<0.5$ \\
\hline $\mathrm{J} 12$ & Furniture textiles & Quilt & $<0.5$ & 24.6 & $<0.5$ & $<0.5$ \\
\hline T13 & Carpets/mats & Sheep skin & $<0.5$ & $<0.5$ & $<0.5$ & $<0.5$ \\
\hline $\mathrm{T} 17$ & Carpets/mats & Carpet & $<0.5$ & $<0.5$ & 0.9 & $<0.5$ \\
\hline T18 & Carpets/mats & Carpet & $<0.5$ & $<0.5$ & $<0.5$ & $<0.5$ \\
\hline T19 & Carpets/mats & Carpet & $<0.5$ & 65.1 & 1.7 & $<0.5$ \\
\hline $\mathrm{T} 20$ & Carpets/mats & Sheep skin & 0.71 & 25.2 & 0.9 & $<0.5$ \\
\hline $\mathrm{T} 21$ & Carpets/mats & Rug & $<0.5$ & 22.3 & 1.4 & $<0.5$ \\
\hline J5 & Carpets/mats & Lamb skin & $<0.5$ & 57.0 & $<0.5$ & $<0.5$ \\
\hline J9 & Carpets/mats & Bath mat & $<0.5$ & 74.4 & $<0.5$ & $<0.5$ \\
\hline J11 & Carpets/mats & Bath mat & $<0.5$ & 59.1 & $<0.5$ & $<0.5$ \\
\hline T14 & Cotton/leather clothes & Jacket & $<0.5$ & $<0.5$ & $<0.5$ & $<0.5$ \\
\hline T15 & Cotton/leather clothes & Jacket & $<0.5$ & 0.71 & 0.6 & $<0.5$ \\
\hline T16 & Cotton/leather clothes & Jacket & $<0.5$ & $<0.5$ & $<0.5$ & $<0.5$ \\
\hline $\mathrm{J} 2$ & Cotton/leather clothes & Bath robe & $<0.5$ & 373 & $<0.5$ & $<0.5$ \\
\hline $\mathrm{T} 25$ & Food contact material & Baking mold & $<0.5$ & $<0.5$ & $<0.5$ & $<0.5$ \\
\hline T26 & Food contact material & Paper plates & $<0.5$ & $<0.5$ & 12.3 & 3.29 \\
\hline $\mathrm{T} 27$ & Food contact material & Baking cover & $<0.5$ & $<0.5$ & $<0.5$ & $<0.5$ \\
\hline $\mathrm{T} 28$ & Food contact material & Paper plates & $<0.5$ & $<0.5$ & 1.2 & $<0.5$ \\
\hline $\mathrm{T} 29$ & Food contact material & Paper cup & $<0.5$ & $<0.5$ & 0.6 & $<0.5$ \\
\hline
\end{tabular}

Concentrations above MDL are presented in bold.

Washburn et al., 2005; Mawn et al., 2005; Larsen et al., 2006; Russell et al., 2008, 2010) and market screening studies where samples have been collected without detailed knowledge about the chemistry being used in the sampled products (Herzke et al., 2012; Greenpeace, 2013; Brigden et al., 2013; Knepper et al., 2014; Liu et al., 2014). Table 1 demonstrates that extractable concentrations of PFASs display considerable variability, ranging over five orders of magnitude for individual homologues between the different studies. Although there's some overlap in the range of detected concentrations, it can be noted that more recently sampled products (2006-2013) contain substantially lower concentrations of PFOA and 8:2 FTOH compared to similar products sampled prior to 2006 (Washburn et al., 2005; Berger and Herzke, 2006). Furthermore, the concentrations of PFOS residuals in textile and carpet samples from recent studies were approximately 5 orders of magnitude lower compared to similar products sampled prior to the phase out of PFOS-related chemistry by 3M in 2000-2002 (Fraunhofer, 2004). Since the fluorochemical industry has made several commitments to replace long-chain PFAS with short-chain alternatives and reduce residuals in finished products (USEPA, 2000; USEPA, 2006), the differences between studies can most likely be attributed to temporal changes in the use of PFASs in textile and carpet treatment processes and industry efforts to remove low molecular weight PFAS residuals from finished products (USEPA, 2006). This explanation is also supported by Liu et al. (2014) who observed a statistically significant decrease in PFOA residuals in several consumer product categories in the US between 2007 and 2011. It may also be noted in this context that the products imported from China (which has ongoing production of PFOS and related chemicals) do not have notably different composition of PFASs in products sampled randomly from the market in recent years. The high detection frequency of 6:2 FTOH in this study rather implies that the textile treatment solutions used in Chinese textiles are primarily based on short-chain PFASs.

Although there is indicative evidence that PFAS concentrations in consumer products have decreased due to industry actions, differences in sampling and analysis may also explain some of the variability between different studies (Table 1). To begin with, the lack of a stringent sample definition for treated consumer products makes it difficult to know if the analyzed samples are representative of the product categories present on the market. In contrast to the studies performed by industry (Fraunhofer, 2004; Washburn et al., 2005; Mawn et al., 2005; Larsen et al., 2006; Russell et al., 2008, 2010), market screening studies have limited information about how the samples were treated and handled prior to the date of purchase. Differences in sub-sampling may also affect the quantitative results. For instance, some studies report the presence of PFASs in the treated carpet fibers (Washburn et al., 2005), whereas this and other studies (Herzke et al., 2012; Liu et al., 2014) also analyzed the carpet backing. Repeated measurements of PFAS residuals in well-defined fluorochemical raw materials and finished products may also display some variability due to the heterogeneity of the samples (Fraunhofer, 2004; Larsen et al., 2006; Liu et al., 2014). For measurements of FTOHs using GC-MS it has also been hypothesized that fragmentation of co-extracted polymeric PFASs in the GC inlet may lead to an overestimation of the extractable amount of residuals (Larsen et al., 2006). However, we did not test for this since no information about the applied fluoropolymers in the investigated products was available to us. Given the efforts to regulate PFOS (UNEP, 2009) and more recently PFOA in finished products there's clearly a need for robust and standardized methods for sampling and analysis. Inter-laboratory comparison studies (Weiss et al., 2013) would also be valuable to evaluate and improve the reproducibility of analytical data for PFASs in various consumer products.

\subsection{Estimated emissions of PFASs to air and water from imported consumer products}

The population-normalized emission rates of imported products from China were estimated to be 6.59, 2130 and $197 \mu$ year $^{-1}$ capita $^{-1}$ for PFOA, 6:2 FTOH and 8:2 FTOH respectively for the intermediate scenario based on Norwegian import data. A large range of uncertainty in estimated emission rates (spanning 2-3 orders of magnitude) was observed for all the homologues due the variable concentrations in consumer product samples (Fig. 1). To put the estimated emissions from Chineseimported consumer products into perspective, a comparison with total diffuse emissions to municipal wastewater influent in Sweden (Filipovic et al., 2013, 2014) and the total off-gassing of neutral PFASs from the city of Zurich (Wang et al., 2012) is presented in Fig. 1. The intermediate population normalized emission rates of PFOA from Chinese-imported consumer products were approximately a factor of 67 lower than those estimated to be discharged in 
Table 3

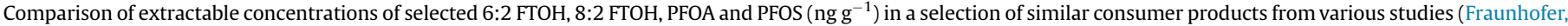
2004; Washburn et al., 2005; Berger and Herzke, 2006; Herzke et al., 2012: Liu et al., 2014; Knepper et al., 2014).

\begin{tabular}{|c|c|c|c|c|c|c|c|}
\hline \multicolumn{8}{|c|}{ Concentration of extractable PFASs $\left(\mathrm{ng} \mathrm{g}^{-1}\right)$} \\
\hline Sample & $\mathrm{n}$ & $6: 2 \mathrm{FTOH}$ & $8: 2 \mathrm{FTOH}$ & PFOA & PFOS & Sampling year & Ref. \\
\hline Treated fabric web & 2 & NA & NA & NA & $<5000-610000$ & 2001 & Fraunhofer 2004 \\
\hline Mill-treated carpet & $>60$ & NA & NA & $200-600$ & NA & 2004 & Washburn et al., 2005 \\
\hline Treated apparel & $>100$ & NA & NA & $<$ MDL-1400 & NA & 2004 & Washburn et al., 2005 \\
\hline Treated upholstery & 3 & NA & NA & $<34$ & NA & 2004 & Washburn et al., 2005 \\
\hline Outdoor clothing & 15 & $<14-110$ & $<14-123006$ & $2-211$ & $<0.1-356$ & 2006 & Berger and Herzke 2006 \\
\hline Pre-treated carpeting & 9 & $\mathrm{NA}$ & NA & $<$ MDL-226 & $<\mathrm{MDL}$ & $2007-2011$ & Liu et al., 2014 \\
\hline Treated apparel & 13 & NA & NA & $<$ MDL-269 & $<\mathrm{MDL}$ & $2007-2010$ & Liu et al., 2014 \\
\hline Treated home textiles and upholstery & 6 & NA & NA & $19-330$ & $<\mathrm{MDL}$ & $2007-2011$ & Liu et al., 2014 \\
\hline Treated non-voven medical garments & 9 & NA & NA & $<$ MDL-369 & $<\mathrm{MDL}$ & $2008-2011$ & Liu et al., 2014 \\
\hline Food contact material & 9 & NA & NA & $1.8-2500$ & $<\mathrm{MDL}$ & $2007-2011$ & Liu et al., 2014 \\
\hline Membranes for apparel & 8 & NA & NA & $<$ MDL-163 & $<$ MDL-8.6 & $2007-2011$ & Liu et al., 2014 \\
\hline Carpets & 2 & $14-183$ & $183-307$ & $<$ MDL-1.4 & $0.6-0.9$ & 2012 & Herzke et al., 2012 \\
\hline Textiles & 2 & $36-128$ & $509-840$ & $2.7-25$ & $<\mathrm{MDL}$ & 2012 & Herzke et al., 2012 \\
\hline Leather & 2 & $<\mathrm{MDL}$ & $22-385$ & $<\mathrm{MDL}$ & $35-63$ & 2012 & Herzke et al., 2012 \\
\hline Food contact material & 2 & $<\mathrm{MDL}$ & $<\mathrm{MDL}$ & $<\mathrm{MDL}$ & $<\mathrm{MDL}$ & 2012 & Herzke et al., 2012 \\
\hline Outdoor clothing & 7 & $<$ MDL-380 & $<$ MDL-5100 & $<$ MDL-7.4 & $<\mathrm{MDL}-4.46$ & 2012 & Brigden et al., 2013 \\
\hline Outdoor clothing & 17 & $<$ MDL-7200 & $<$ MDL-6600 & $<$ MDL-9.8 & $<$ MDL-33.2 & 2012 & Greenpeace 2013 \\
\hline Outdoor clothing & 15 & $<$ MDL-124 & $<\mathrm{MDL}-3440^{\mathrm{a}}$ & $<$ MDL-30.6 & $<$ MDL-0.33 & $2011-2012$ & Knepper et al., 2014 \\
\hline Furniture textiles & 27 & $<$ MDL-1210 & $<$ MDL-1000 & $<$ MDL-5.6 & $<\mathrm{MDL}$ & 2013 & This study \\
\hline Carpets, mats & 9 & $<$ MDL-62 & $<$ MDL-1.6 & $<$ MDL-0.17 & $<$ MDL-0.79 & 2013 & This study \\
\hline Cotton and leather clothes & 4 & $<$ MDL-2270 & $<$ MDL-1.42 & $<$ MDL-0.18 & $<\mathrm{MDL}$ & 2013 & This study \\
\hline Food contact material & 5 & $<\mathrm{MDL}$ & $>$ MDL-1.8 & $<\mathrm{MDL}$ & $<\mathrm{MDL}$ & 2013 & This study \\
\hline
\end{tabular}

${ }^{\text {a }}$ Concentrations reported in $\mu \mathrm{g} \mathrm{m}^{-2}$ were transformed to $\mathrm{ng} \mathrm{g}^{-1}$ using an average weight of outdoor clothing textiles of $1.5 \mathrm{~g}$ dm ${ }^{-2}$ derived from Berger and Herzke (2006).

municipal wastewater influents after correcting for background contamination in tap water (Filipovic and Berger, 2014). For 8:2 FTOH the intermediate population normalized emission rates estimated in this study were a factor 300 lower than the estimated total diffuse emissions in the city of Zurich (Wang et al., 2012). Unfortunately, a comparison with total diffuse emissions was not possible for 6:2 FTOH, since these were not calculated by Wang et al. (2012).

The lack of agreement between different emission estimation approaches (Fig. 1) suggests that emissions from imported consumer products, as calculated in this study, would have a negligible impact on the total diffuse emissions. This result is surprising since previous studies have suggested that treated textiles and carpets are the major diffuse sources of PFOA and 8:2 FTOH to water and air respectively (Buser and Morf, 2009). A comparison with the input data used by Buser and Morf (2009) reveal that total import numbers of treated textiles are slightly lower than this study (3.2 compared to $7.5 \mathrm{~kg} \mathrm{year}^{-1}$ capita $^{-1}$ ) whereas extractable concentrations of PFOA and 8:2 FTOH are 2-4 orders of magnitude higher than used in this study for the intermediate scenario. The discrepancy in emission estimates between this study and that of Buser and Morf (2009) can therefore be attributed to the relatively low concentrations of extractable PFASs observed in this study (Table 1). Combined with the preliminary evidence of a decreasing trend in PFAS concentrations in consumer products over time (Liu et al., 2014), the discrepancy in emission estimates may therefore suggest that urban diffuse releases to air and water (as estimated by Wang et al., 2012; Filipovic et al., 2013) reflect a legacy effect of old

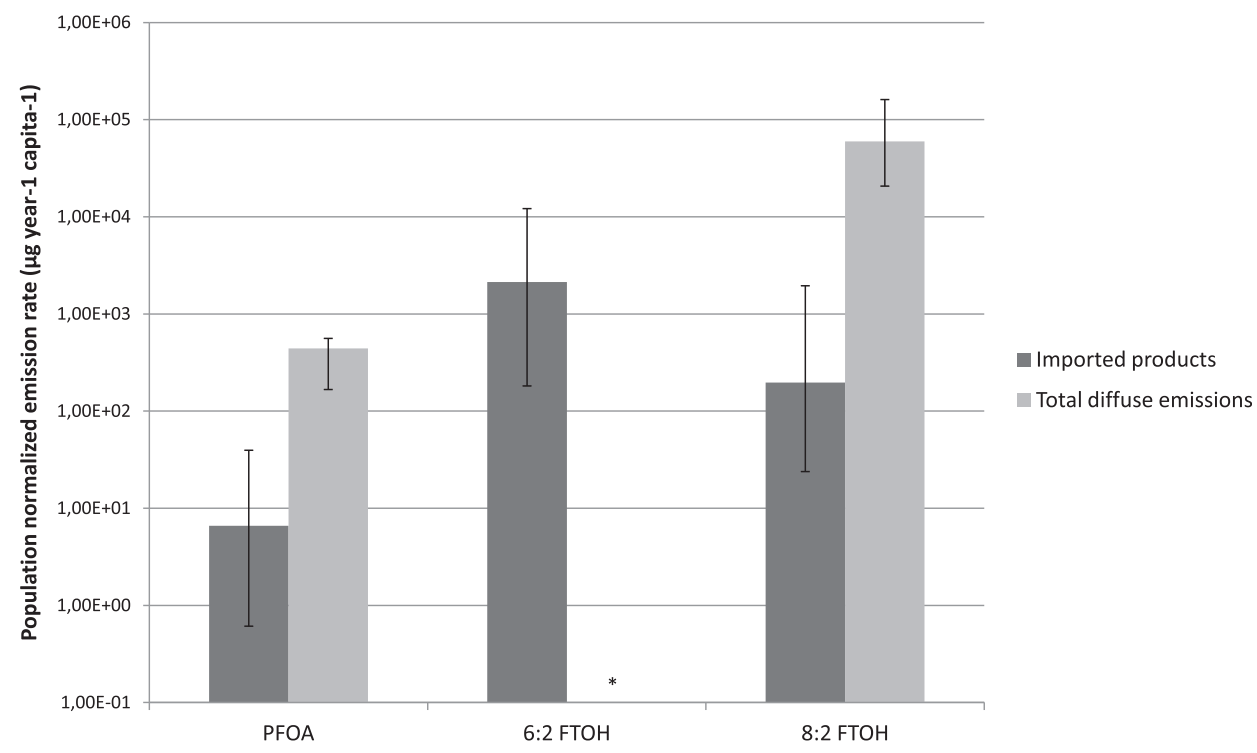

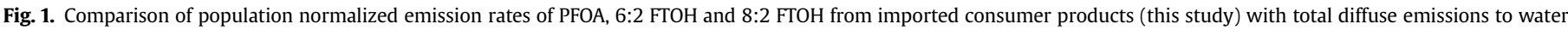

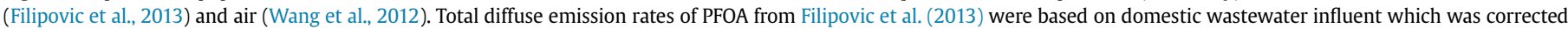
for the background concentration in tap water. *Not calculated by Wang et al. (2012). 
product emissions. For emissions of FTOHs to air, the releases occurring during disposal (e.g. landfill and incineration) (Weinberg et al., 2011a,b) should also be considered for a more representative comparison with Wang et al. (2012).

Another factor to consider is that textiles, carpets and food contact materials from other countries than China will add to the emissions estimated here. However, since the amount of textiles imported from China makes up approximately $40 \%$ of the total import of this product category (SSB, 2008) this could not explain the disagreement between emission estimates in Fig. 1. It is also possible that PFAS residuals in consumer products not considered here, including personal care products (Fujii et al., 2013) and textile treatment solutions (Herzke et al., 2012; Knepper et al., 2014; Fiedler et al., 2011; Liu et al., 2014), make a significant contribution to the total diffuse emissions of PFASs.

\section{Conclusions and future directions}

This study demonstrates that extractable PFASs are present in a wide range of consumer products which are imported from China to Norway in large quantities. However, the concentrations measured here were lower than those reported in historically used products and the majority of consumer products imported from China comply with international regulations (UNEP, 2009; ECHA, 2013). Based on the comparison with total per capita emission rates, we conclude that the emissions from consumer products imported from China account for 1.5 per cent of the discharges of PFOA to wastewater influents and 0.3 per cent of the emissions of 8:2 FTOH to air in the intermediate emission scenario using Norwegian import data. When using the data from the high emission scenario the relative importance of consumer product emissions increases to 9 and 3 per cent of the total per capita emissions for PFOA and FTOHs respectively. Thus, despite the uncertainty in extractable PFAS concentrations and emission factors we find no support for the hypothesis that diffuse emissions from imported consumer products would be a major ongoing source of PFASs to the environment in non-producing countries such as Norway.

A remaining caveat of constraining diffuse emissions from consumer products is the large variability in extractable PFAS residuals reported in different studies. Although some of this variability can be attributed to a decreasing temporal trend (Liu et al., 2014), biases in the collection of consumer product samples and differences in analytical methods cannot be ruled out. Further work should therefore be devoted to establish robust sampling and analysis protocols for PFASs in consumer product samples including inter-laboratory comparison studies.

\section{Acknowledgments}

This work received financial support from the Norwegian Research Council via research project PFC ChiNo (29666/E40). Additional funding was also provided by the External Cooperation Program of Chinese Academy of Sciences (GJHZ1202). Therese Haugdal Nøst and Børge Moe are gratefully acknowledged for their assistance with the sampling campaign.

\section{Appendix A. Supplementary data}

Supplementary data related to this article can be found at http:// dx.doi.org/10.1016/j.envpol.2014.12.034.

\section{References}

3 M Company, 2000. Voluntary Use and Exposure Information Profile for Perfluorooctanoic Acid and Salts. USEPA Administrative Record. AR226-0595.
Available from: www.regulations.gov. as document EPA-HQ-OPPT-2002-00510009

Begley, T.H., White, K., Honigfort, P., Twaroski, M.L., Neches, R., Walker, R.A., 2005. Perfluorochemicals: potential sources of and migration from food packaging. Food. Addit. Contam. 22, 1023-1031.

Berger, U., Herzke, D., 2006. Per- and polyfluorinated alkyl substances (PFAS) extracted from textile samples. Organohalogen Compd. 68, 2023-2026.

Brigden, K., Hetherington, S., Wang, M., Santillo, D., Johnston, P., 2013. Hazardous Chemicals in Branded Textile Products on Sale in 25 Countries/regions During 2013. Greenpeace Research Laboratories Technical Report 06/2013, available from: http://www.greenpeace.org/international/Global/international/ publications/toxics/2014/Technical-Report-01-2014.pdf

Buck, R.C. Franklin, J., Berger, U., Conder J.M., Cousins, I.T, De Voogt, P., Jensen, A.A. Kannan, K., Mabury, S.A., Van Leeuwen, S.P.J., 2011. Perfluoroalkyl and polyfluoroalkyl substances in the environment: terminology, classification and origins. Integr. Environ. Assess. Manag. 7, 513-541.

Buser, A.M., Morf, L., 2009. Substance Flow Analysis for Switzerland Perfluorinated Surfactants Perfluorooctanesulfonate (PFOS) and Perfluorooctanoic Acid (PFOA). Federal Office for the Environment (FOEN), 22/09, Bern. www. environment-switzerland.ch/uw-0922-e.

Conder, J.M., Hoke, R.A., de Wolf, W., Russell, M.H., Buck, R.C., 2008. Are PFCAs bioaccumulative? A critical review and comparison with regulatory criteria and persistent lipophilic compounds. Environ. Sci. Technol. 42, 995-1003.

Dinglasan-Panlilio, M.J.A., Mabury, S.A., 2006. Significant residual fluorinated alcohols present in various fluorinated materials. Environ. Sci. Technol. 40, 1447-1453.

ECHA, 2013. Agreement of the member state committee on the identification of perfluorooctanoic acid (PFOA) as a substance of very high concern. http://echa. europa.eu/documents/10162/14598347/agreement_pfoa_20130614_en.pdf (adopted on 14.06.13.).

Fiedler, S., Pfister, G., Schramm, K.-W., 2011. Poly- and perfluorinated compounds in household consumer products. Toxicol. Environ. Chem. 92, 1801-1811.

Filipovic, M., Berger, U., 2014. Are perfluoroalkyl acids in waste water treatment plant effluents the result of primary emissions from the technosphere or of environmental recirculation? Chemosphere. DOI: 10.1016.

Filipovic, M., Berger, U., McLachlan, M.S., 2013. Mass balance of perfluoroalkyl acids in the Baltic Sea. Environ. Sci. Technol. 47, 4088-4095.

Fraunhofer, 2004. Fraunhofer Institute of Toxicology and Experimental Medicine (ITEM). Appendix A. Test sample re-analysis (performed by 3M). In: Final Report: 28-Day Repeated Dermal Contact Study of 3M Test Articles in SpragueDawley Rats. Fraunhofer ITEM. Study No.: 01G 000 02. AR226-1874a.

Fujii, Y., Harada, K.H., Koizumi, A., 2013. Occurence of perfluorinated carboxylic acids (PFCAs) in personal care products and compounding agents. Chemosphere 93, 538-544.

Gewurtz, S.B., Bhavsar, S.P., Crozier, P.W., Diamond, M.L., Helm, P.A., Marvin, C.H., Reiner, E.J., 2009. Perfluoroalkyl contaminants in window film: indoor/outdoor, urban/rural, and winter/summer contamination and assessment of carpet as a possible source. Environ. Sci. Technol. 43, 7317-7323.

Greenpeace, 2013. Chemistry for Any Weather. Available from: http://www. greenpeace.de/sites/www.greenpeace.de/files/publications/20120315-outdoorreport-engl.pdf.

Herzke, D., Olsson, E., Posner, S., 2012. Perfluoroalkyl and polyfluoroalkyl substances (PFASs) in consumer products in Norway - a pilot study. Chemosphere 88, 980-987.

Kissa, E., 2001. Fluorinated Surfactants and Repellents (2nd edition revised and expanded). In: Surfactant Science Series 97. Marcel Dekker, New York (NY).

Knepper, T.P., Frömel, T., Gremmel, C., van Driezum, I., Weil, H., Vestergren, R., Cousins, I., 2014. Understanding the Exposure Pathways of Per- and Polyfluoralkyl Substances (PFASs) Via Use of PFASs-containing Products - Risk Estimation for Man and Environment. Report to the Federal Ministry for the Environment, Nature Conservation and Nuclear Safety UFOPLAN (Main Task): Substance Risks Project No. (FKZ) (3711 63 418).

Larsen, B.S., Stchur, P., Szostek, B., Bachmura, S.F., Rowand, R.C., Prickett, K.B., Korzeniowski, S.H., Buck, R.C., 2006. Method development for the determination of residual fluorotelomer raw materials and perflurooctanoate in fluorotelomer-based products by gas chromatography and liquid chromatography mass spectrometry. J. Chromatogr. A 1110, 117-124.

Lau, C., Anitole, K., Hodes, C., Lai, D., Pftales-Hutchens, A., Seed, J., 2007. Perfluoroalkyl acids: a review of monitoring and toxicological findings. Toxicol. Sci. 99, 366-394.

Lim, T.C., Wang, B., Huang, J., Deng, S., Yu, G., 2011. Emission inventory for PFOS in China: review of past methodologies and suggestions. Sci. World J. 11, 1963-1980.

Lindstrom, A.B., Strynar, M.J., Libelo, E.L., 2011. Polyfluorinated compounds: past, present, and future. Environ. Sci. Technol. 75, 7954-7961.

Liu, X., Guo, Z., Krebs, K.A., Pope, R.A., Roache, N.F., 2014. Concentrations and trends of perfluorinated chemicals in potential indoor sources from 2007 through 2011 in the US. Chemosphere 98, 51-57.

Mawn, M.P., McKay, R.G., Ryan, T.W., Szostek, B., Powley, C.R., Buck, R.C., 2005 Determination of extractable perfluorooctanoic acid (PFOA) in water, sweat simulant, saliva simulant, and methanol from textile and carpet samples by LC/ MS/MS. Analyst 130, 670-678.

Natarajana, R., Azerad, R., Badet, R., Copin, E., 2005. Microbial cleavage of C-F bond. J. Fluor. Chem. 126, 425-436.

Norin, H., Schulze, P.E., 2007. Fluorerade miljogifter i impregneringsmedel 
Published. Naturskyddsforeningen. Publisher: 20077971.

Paul, A.G., Jones, K.C., Sweetman, A.J., 2009. A first global production, emission and environmental inventory for perfluorooctane sulfonate. Environ. Sci. Technol. 43, 386-392.

Powley, C., George, S.W., Ryan, T.W., Buck, R.C., 2005. Matrix effect-free analytical methods for determination of perfluorinated carboxylic acids in environmental matrixes. Anal. Chem. 77, 6353.

Prevedouros, K., Cousins, I.T., Buck, R.C., Korzeniowski, S.H., 2006. Sources, fate and transport of perfluorocarboxylates. Environ. Sci. Technol. 40, 32-44.

Russell, M.H., Berti, W.R., Szostek, B., Buck, R.C., 2008. Investigation of the biodegradation potential of a fluoroacrylate polymer product in aerobic soils. Environ. Sci. Technol. 42, 800-807.

Russell, M.H., Berti, W.R., Szostek, B., Wang, N., Buck, R.C., 2010, Evaluation of PFO formation from the biodegradation of a fluorotelomer-based urethane polymer product in aerobic soils. Polym. Degrad. Stab. 95, 79-85.

Schlummer, M., Gruber, L., Fiedler, D., Kizlauskasa, M., Müller, J., 2013. Detection of fluorotelomer alcohols in indoor environments and their relevance for human exposure. Environ. Int. 57-58, 42-49.

SFT, 2006. Kartlegging av perfluoralkylstoffer (PFAS) i utvalgte tekstiler Published.

SSB, 2008. Importpris med kinaeffekt-en importprisligning for tekstil och bekledningsprodukter (Pl18) (cited 2014 May 25). Available from: http://www.ssb.no/ a/publikasjoner/pdf/notat_200848/notat_200848.pdf (in Norwegian).

SSB, 2013. Norwegian Statistics on External Trade in Goods (Cited 2013 May 25). Available from: https://www.ssb.no/statistikkbanken/selecttable/hovedtabell Hjem.asp?KortNavnWeb=muh\&CMSSubjectArea $=$ utenriksokonomi\& PLanguage $=1 \&$ checked $=$ true.

Trier, X., Granby, K., Christiansen, C.H., 2011. Polyfluorinated surfactants (PFS) in paper and board coatings for food packaging. Environ. Sci. Pollut. Res. 18, 1108-1120.

UNEP, 2009. United Nations Environment Programme. Governments Unite to Stepup Reduction on Global DDT Reliance and Add Nine New Chemicals Under International Treaty (cited 2010 December 11). Available from: http://chm.pops. int/Convention/PressreleaseCOP4Geneva8May2009/tabid/542/language/en-US/
Default.aspx.

USEPA, 2000. US Environmental Protection Agency. EPA and 3M Announce Phaseout of PFOS (cited 2014 January 10). Available from: http://yosemite.epa. gov/opa/admpress.nsf/0/33aa946e6cb11f35852568e1005246b4.

USEPA, 2006. US Environmental Protection Agency. 2010/2015 PFOA Stewardship Program [cited 2014 January 10]. Available from: http://www.epa.gov/opptintr/ pfoa/pubs/stewardship/.

Wang, Z.Y., Scheringer, M., MacLeod, M., Bogdal, C., Mueller, C.E., Gerecke, A.C. Hungerbuehler, K., 2012. Atmospheric fate of poly- and perfluorinated alkyl substances (PFASs): II. Emission source strength in summer in Zurich. Switz. Environ. Pollut. 169, 204-209.

Wang, Z., Cousins, I.T., Scheringer, M., Hungerbuehler, K., 2013. Fluorinated alternatives to long-chain perfluoroalkyl carboxylic acids (PFCAs), perfluoroalkane sulfonic acids (PFSAs) and their potential precursors. Environ. Int. 60, 242-248.

Wang, Z., Cousins, I.T., Scheringer, M., Buck, R.C., Hungerbuehler, K., 2014. Globa emission inventories for C4-C14 perfluoroalkylcarboxylic acid (PFCA) homologues from 1951 to 2030, part I: production and emissions from quantifiable sources. Environ. Int. 70, 62-75.

Washburn, S.T., Bingman, T.S., Braithwaite, S.K., Buck, R.C., Buxton, L.W., Clewell, H.J. Haroun, L.A., Kester, J.E., Rickard, R.W. Shipp, A.M., 2005. Exposure assessment and risk characterization for perfluorooctanoate in selected consumer articles. Environ. Sci. Technol. 39, 3904-3910.

Weinberg, I., Dreyer, A., Ebinghaus, R., 2011a. Waste water treatment plants as sources of polyfluorinated compounds, polybrominated diphenyl ethers and musk fragrances to ambient air. Environ. Pollut. 159, 125-132.

Weinberg, I., Dreyer, A., Ebinghaus, R., 2011b. Landfills as sources of polyfluorinated compounds, polybrominated diphenyl ethers and musk fragrances to ambient air. Atmos. Environ. 45, 935-941.

Weiss, J.M., Van Der Veen, I., De Boer, J., 2013. Analytical improvements shown over four interlaboratory studies of perfluoroalkyl substances in environmental and food samples. TrAC Trends. Anal. Chem. 43, 204-216.

Xie, S.W., Wang, T.Y., Liu, S.J., Jones, K., Sweetman, A., 2013. Industrial source identification and emission estimation of perfluorooctane sulfonate in China. Environ. Int. 53, 1-8. 